CARDIOVASCULAR MEDICINE

\title{
Evaluation of survival and ischaemic and thromboembolic event rates in patients with non- valvar atrial fibrillation in the general population when treated and untreated with warfarin
}

\author{
C J Currie, M Jones, J Goodfellow, P McEwan, C L Morgan, C Emmas, J R Peters
}

Heart 2006;92:196-200. doi: 10.1136/hrt.2004.058339

See end of article for authors' affiliations .....................

Correspondence to: Dr Craig Currie, Cardiff Research Consortium, Heath Park, Cardiff CF14 4UJ, UK; curriec@cardiff. ac.uk

Accepted 20 April 2005 Published Online First 12 May 2005

\begin{abstract}
Objective: To compare survival and adverse outcome of patients with non-valvar atrial fibrillation (NVAF) treated with or without warfarin.

Design: Record linkage method to identify patients with a previous hospital diagnosis of atrial fibrillation and to link these patients to international normalised ratio (INR) test results and mortality data.

Setting: Cardiff and the Vale of Glamorgan, Wales.

Main outcome measures: Mortality, specifically from ischaemic and thromboembolic events.

Results: 6108 patients were identified with NVAF, of whom $36.4 \%$ received warfarin. Mean survival in the warfarin and non-warfarin groups was 52.0 months and 38.2 months, respectively $(p<0.001)$, and 14.4 months ( $p<0.001$ ) after adjustment for confounding factors. Warfarin treated patients in the upper quartile of INR control had significantly longer survival (57.5 months) than did those in the lowest quartile of control (38.1 months, $p<0.001$ ). The risk of stroke in the warfarin group when treated was lower than that in the non-warfarin group (relative rate (RR) $0.74, p<0.001$ ). The risk of death from ischaemic stroke was lower in the warfarin group (RR 0.43, $p<0.001$ ). The risk of all ischaemic and embolic events in the warfarin group was lower when they were taking warfarin (RR $0.74, p<0.001$ ). The risk of bleeding in the warfarin group when treated was greater (RR 1.78, $p=0.001$ ).

Conclusions: Patients with NVAF within the recommended target INR range of 2.0-3.0 survive longer and have reduced morbidity. Probably too few people are anticoagulated with warfarin in NVAF.
\end{abstract}

$\mathrm{N}$ on-valvar atrial fibrillation (NVAF) affects about $1 \%$ of the general population ${ }^{1}$ with the incidence increasing to $4.7 \%$ in people over the age of 65 years. NVAF is associated with a significant increase in morbidity and mortality, with a three- to fivefold increased risk of stroke. ${ }^{23}$

Randomised clinical trials have shown that anticoagulation with warfarin reduces the risk of stroke by up to two thirds. ${ }^{4-}$ ${ }^{10}$ However, fewer than half of patients eligible for warfarin treatment actually receive it. ${ }^{11-12}$ Recent national initiatives such as the National Strategic Framework for the Elderly have included standards that aim at improving the proportion of NVAF patients at risk of stroke who receive anticoagulation..$^{13} 14$

The pharmacokinetic profile of warfarin is complex ${ }^{9}$ and frequent monitoring is required to maintain patients with NVAF within the recommended international normalised ratio (INR) target range of 2.0-3.0. Mortality is increased among patients with NVAF who are outside the target range. ${ }^{14}$ Among patients treated with warfarin who are below the target range at the time of a stroke event, severity is greater and 30 day survival is reduced compared with patients at an INR greater than 2.0. ${ }^{15}$ Many factors have been reported as barriers to treatment with warfarin, including interactions between warfarin and other medications and foods, concerns over the risk of bleeding, and practical problems relating to frequent INR monitoring. ${ }^{16}$

The purpose of this study was to characterise the epidemiology of serious thromboembolic and bleeding events and mortality among patients with NVAF who were anticoagulated with warfarin compared with those who were not anticoagulated with warfarin in a large UK general population.

\section{METHODS}

\section{Data sources}

As has been described previously, ${ }^{17}$ three sets of routinely collected data were used: inpatient data from April 1995 to March 2000 containing a range of information including discharge diagnosis (International classification of diseases, 10th revision (ICD-10)), length of stay, and operative procedures (Classification of surgical operations and procedures, fourth revision); haematology laboratory reports dating from June 1995 to January 2002 containing details of INR monitoring test results (including any initiated by primary care providers); and mortality data derived from the Office of National Statistics from January 1995. These data records were linked both within and between the various data sets by probability matching algorithms to identify those records relating to the same patient. ${ }^{18}$

\section{Participants}

The study was conducted in Cardiff and the Vale of Glamorgan, a largely urban area of South Wales, UK, with a population of 424000 . The patients studied were those identified during the study period with a diagnosis of atrial fibrillation (AF) (ICD-10 rubric I48 $^{*}$ ) recorded on inpatient admission and with no record of heart valve procedures before AF. Patients with more than four INR recordings before or after their initial NVAF admission were assumed to have received oral anticoagulation in the form of warfarin. These patients constituted the warfarin group. We assumed

Abbreviations: AF, atrial fibrillation; ICD-10, International classification of diseases, 10th revision; INR, international normalised ratio; NVAF, non-valvar atrial fibrillation; RR, relative rate 
that the remaining patients, the non-warfarin group, had not been maintained on warfarin.

\section{Linear interpolation of INR values}

To estimate the daily INR values between routine measurements, we assumed that the actual difference in INR between any two consecutive measurements was linear, and the data were interpolated accordingly. ${ }^{19}$ The level of coagulation control for each patient was assessed by the percentage of interpolated INR values within the target range of 2.0-3.0.

\section{Selection and coding of clinical outcomes}

Serious cardiovascular events, including acute myocardial infarction, pulmonary embolism, deep vein thrombosis, ischaemic stroke, and bleeding events, were characterised. Events were selected as being a primary diagnosis only on inpatient admissions; however, an event was recorded as such if a selected code was anywhere on the death record.

The epidemiology of stroke ${ }^{20}$ and heart disease $\mathrm{e}^{21}$ has been described previously in the same population by similar methods.

\section{Statistical analyses}

The time origin for these analyses was the first identifiable inpatient diagnosis of AF. In addition to this index date for all patients, a second time origin was necessary for the group who received warfarin because they were not necessarily anticoagulated with warfarin immediately after the index diagnosis of AF. This allowed statistical comparisons to be made between the warfarin group when treated versus the non-warfarin group, as well as comparisons of the rate of events within the warfarin treated group while treated and

Table 1 Rates of the serious adverse events among patients with non-valvar atrial fibrillation when treated or untreated with warfarin

\begin{tabular}{|c|c|c|c|c|c|c|c|}
\hline & \multicolumn{7}{|c|}{ Age category (years) } \\
\hline & $<60$ & $60-64$ & $65-70$ & 71-75 & $76-80$ & $>80$ & All \\
\hline \multicolumn{8}{|c|}{ Number of patients } \\
\hline W & 228 & 203 & 380 & 457 & 465 & 489 & 2223 \\
\hline NW & 301 & 179 & 368 & 486 & 677 & 1860 & 3885 \\
\hline \multicolumn{8}{|c|}{ Observation period (years) } \\
\hline $\mathrm{W}_{+}$ & 392 & 345 & 634 & 688 & 610 & 587 & 3257 \\
\hline W- & 183 & 127 & 226 & 289 & 278 & 297 & 1400 \\
\hline NW & 660 & 335 & 701 & 859 & 1086 & 2651 & 6309 \\
\hline \multicolumn{8}{|c|}{ Acute myocardial infarction } \\
\hline$W_{+}$ & 0.76 & 2.61 & 1.74 & 2.61 & 2.62 & 3.92 & 2.46 \\
\hline W- & 0.55 & 3.14 & 2.66 & 3.80 & 1.08 & 2.36 & 2.29 \\
\hline NW & 1.36 & 1.49 & 2.85 & 3.49 & 5.26 & 3.35 & 3.66 \\
\hline \multicolumn{8}{|c|}{ Pulmonary embolism } \\
\hline$W_{+}$ & 0.00 & 0.29 & 0.32 & 0.58 & 0.66 & 1.36 & 0.58 \\
\hline W- & 0.00 & 0.00 & 1.33 & 1.73 & 0.72 & 2.02 & 1.36 \\
\hline NW & 0.00 & 0.60 & 0.71 & 0.70 & 0.74 & 0.76 & 0.72 \\
\hline \multicolumn{8}{|c|}{ Deep vein thrombosis } \\
\hline$W_{+}$ & 0.00 & 0.00 & 0.16 & 0.15 & 0.49 & 0.68 & 0.28 \\
\hline W- & 0.55 & 0.78 & 0.00 & 1.38 & 0.00 & 0.67 & 0.57 \\
\hline NW & 0.00 & 0.00 & 0.00 & 0.10 & 0.00 & 0.16 & 0.10 \\
\hline \multicolumn{8}{|c|}{ Ischaemic stroke } \\
\hline$W_{+}$ & 1.02 & 2.03 & 3.63 & 5.08 & 5.08 & 8.52 & 4.61 \\
\hline W- & 2.19 & 4.71 & 6.21 & 6.22 & 6.48 & 9.76 & 6.36 \\
\hline NW & 0.91 & 1.79 & 3.42 & 5.59 & 5.10 & 8.08 & 6.23 \\
\hline \multicolumn{8}{|c|}{ All bleeding events } \\
\hline W+ & 0.76 & 2.32 & 2.37 & 2.76 & 3.11 & 4.43 & 2.76 \\
\hline W- & 1.09 & 0.00 & 1.33 & 2.07 & 0.72 & 0.67 & 1.14 \\
\hline NW & 0.30 & 3.58 & 1.85 & 1.98 & 0.99 & 1.35 & 1.54 \\
\hline \multicolumn{8}{|c|}{ All ischaemic and embolic events } \\
\hline$W_{+}$ & 1.78 & 4.93 & 5.84 & 8.43 & 8.85 & 14.49 & 7.92 \\
\hline W- & 3.28 & 8.63 & 10.20 & 13.14 & 8.28 & 14.81 & 10.36 \\
\hline NW & 2.27 & 3.88 & 6.99 & 9.89 & 11.10 & 12.36 & 10.69 \\
\hline
\end{tabular}

Rates are of events per 100 person years of observation. NW, non-warfarin group, W, warfarin group; $W+$, warfarin group with treatment; $\mathrm{W}-$, warfarin group without treatment. untreated. A key assumption was that a patient in the warfarin group was considered to be untreated with warfarin if the clinical event occurred before the first INR reading or longer than 42 days after the final recorded INR reading (95\% of INR readings were taken within 42 days of the previous reading). The effect of this assumption was tested in a sensitivity analysis that limited the period during which patients were assumed to be receiving warfarin to within 21 days of the previous test. Kaplan-Meier and Cox proportional hazards models were applied to characterise survival. The Cox proportional hazards models were adjusted for age, sex, and relative morbidity at baseline. The total number of days spent in hospital in the year before the index AF diagnosis was used as a proxy measure of morbidity at baseline. If a patient had one of the quantified events at death, this event was listed among the event rates in tables 1 and 2. Mean life expectancy is detailed rather than median because some of the survival curves did not always reach the 50th centile within the maximum five year window. Event rates were compared by multivariate logistic or Poisson regression models. For the comparison of event rates in the warfarin group when treated and not treated, the non-parametric sign test was applied.

\section{RESULTS}

\section{Identification of the NVAF study groups}

Of 222398 patients hospitalised during the study period 6247 patients with AF were identified. Of these 139 (2.2\%) patients were excluded on the basis of a history of heart valve surgery; the remaining 6108 patients constituted the NVAF study group. Of the NVAF study group 2828 (46.3\%) had an INR reading, with 2223 (36.4\%) having five or more INR readings (the warfarin group). The 3885 (63.6\%) patients with fewer than five INR readings were assumed to be untreated with warfarin (the non-warfarin group). Demographic characteristics differed between the warfarin and non-warfarin groups. The proportion of women was slightly lower in the warfarin group $(46.9 \% \vee 55.4 \%)$. The elderly (age $>75$ years) were less likely than younger patients $(49 \%)$ to be treated with warfarin $(27 \%)$; thus, for both men and women the mean age of warfarin treated patients was lower (men: 69.8 years $v 73.6$ years; women: 75.5 years $v 80.7$ years).

\section{Ischaemic stroke}

There were 631 strokes in the study population; 392 (62\%) strokes occurred in the non-warfarin group and 481 (76\%) strokes occurred when patients, from either group, were not being treated with warfarin. The risk of stroke was lower in the warfarin group when treated (4.61/100 person years) than in the non-warfarin group $(6.23 / 100$ person years, relative rate $(\mathrm{RR}) 0.74, \mathrm{p}<0.001)$. The risk of stroke was

Table 2 Comparison of relative rate of events for ischaemic stroke, thromboembolism, and bleeding in the W+ and NW groups

\begin{tabular}{llrlll}
\hline & \multicolumn{3}{l}{$\begin{array}{l}\text { Events/100 person } \\
\text { years }\end{array}$} & & Crude \\
\cline { 2 - 4 } Event & W+ & NW & $\begin{array}{l}\text { Rdjusted } \\
\text { p value* }\end{array}$ \\
\hline All stroke events & 4.61 & 6.23 & 0.74 & $<0.001 \dagger$ \\
Hospitalised stroke $\neq$ & 2.70 & 2.86 & 0.95 & $0.306 \dagger$ \\
Death from stroke & 2.39 & 5.51 & 0.43 & $<0.001 \S$ \\
All thromboembolic events & 7.92 & 10.69 & 0.74 & $<0.001 \dagger$ \\
Bleeding events & 2.76 & 1.55 & 1.78 & $0.001 \dagger$ \\
\hline
\end{tabular}

*Adjusted for confounding variables, including the duration of the postatrial fibrillation study period; †multivariate Poisson regression; †includes deaths from ischaemic stroke; §multivariate logistic regression.

$\mathrm{RR}$, relative rate. 
also lower in the warfarin group taking treatment (4.61/100 person years) than in the same group not taking treatment (6.36/100 person years, RR $0.72, p<0.001)$. The risk of death from ischaemic stroke was lower in the warfarin group (2.39/ 100 person years $v 5.51 / 100$ person years, RR 0.43, $\mathrm{p}<0.001$ ), with the hospital admission rate for stroke being similar in both groups (2.70/100 person years $v 2.86 / 100$ person years, RR 0.95, $\mathrm{p}=0.308$ ).

\section{Bleeding events}

There were 202 bleeding events in the study population, of which 112 (55\%) occurred when patients, from either group, were not being treated with warfarin. In the warfarin group, the risk of a bleeding resulting in hospitalisation was higher while treated with warfarin $(2.76 / 100$ person years $)$ than in periods of non-treatment (1.07/100 person years, RR 0.39) and higher than in the non-warfarin group (1.54/100 person years, RR 1.78, p =0.001) (table 2). The increased risk of a bleeding event appeared greatest in the oldest patients.

\section{All ischaemic or thromboembolic events}

There were 1076 ischaemic or thromboembolic events, including ischaemic stroke, specifically outlined above, of which $818(76 \%)$ occurred while the patient was not receiving anticoagulation. Patients in the warfarin group who were treated had a significantly lower event rate of 7.92/ 100 person years than did both the same group of patients while not taking warfarin (10.50/100 person years, RR 0.75, $\mathrm{p}<0.001)$ and the non-warfarin group $(10.69 / 100$ person years (RR 0.74, $\mathrm{p}<0.001)$.

\section{Survival}

The mean life expectancy after diagnosis of NVAF was 43.3 months. In a Kaplan-Meier analysis, patients who were treated with warfarin had a mean life expectancy of 52.0 months, whereas those who were not treated with warfarin had a corresponding life expectancy of 38.2 months $(\Delta=13.8$ months, $\mathrm{p}<0.001)$ (fig l). The non-warfarinised group had a high mortality in the first 12 months after AF diagnosis. Subsequent analysis (conditioned on surviving more than 12 months after AF diagnosis) showed that the increased life expectancy in the warfarinised group was maintained $(\mathrm{p}<0.01)$. After age, sex, and morbidity at baseline were added to the Cox proportional hazards model, life expectancy in the warfarinised group increased slightly to 52.6 months, whereas survival in the non-warfarinised group remained at 38.2 months $(\Delta=14.4$ months, $\mathrm{p}<0.001)$. The difference in mortality was preserved in elderly patients older than 75 years, who had an increased life expectancy of

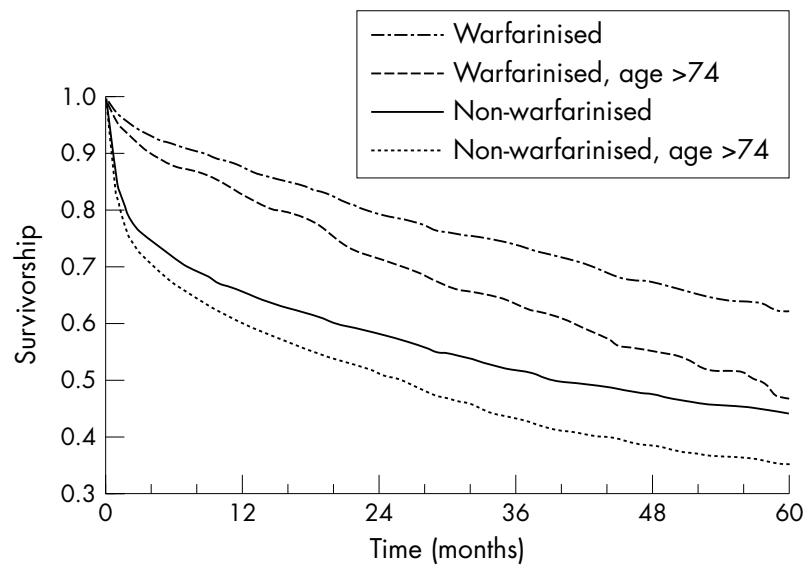

Figure 1 Kaplan-Meier survival analysis of patients with non-valvar atrial fibrillation when treated and untreated with warfarin.

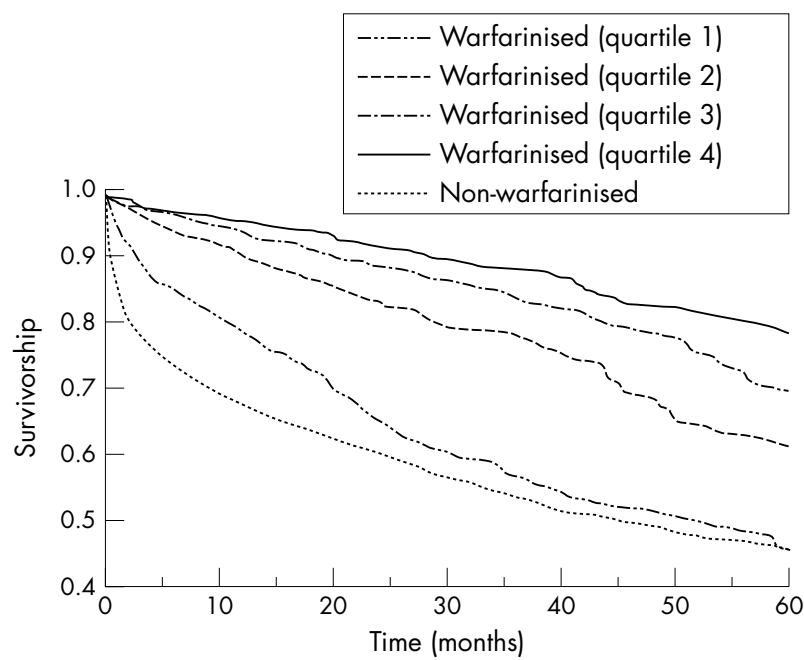

Figure 2 Cox proportional hazards model analysis of survival of patients with non-valvar atrial fibrillation by quartiles of control (proportion of international normalised ratio within target range). The average percentage time in range was $32.4 \%$ in the first quartile, $59.1 \%$ in the second, $71.6 \%$ in the third, and $83.9 \%$ in the fourth.

44.7 months (with warfarin) and 32.5 months (without warfarin; $\Delta=12.2$ months, $\mathrm{p}<0.001$ ).

After dividing patients who received warfarin into quartiles based on their degree of anticoagulation control (percentage of time in target range), a difference in life expectancy was observed. Those in the upper quartile of control (best control), as defined above, had a mean life expectancy of 57.5 months $(\Delta=19.3$ months $v$ non-warfarin treated patients, $\mathrm{p}<0.001)$, whereas those in the lowest quartile of control (worst control) had a mean life expectancy of 38.1 months ( $\Delta=-0.1$ months $v$ non-warfarinised patients, not significant) (fig 2). The difference in life expectancy between the upper and lower quartile of control was 19.4 months $(p<0.001)$.

\section{Sensitivity analysis}

A key assumption in the analyses was that an event that occurred $\geqslant 42$ days after the last INR test was not occurring during warfarin treatment. The effect of changing this to $\geqslant 21$ days was to decrease the number of events in the warfarin treated group when they were classified as being treated with warfarin: ischaemic stroke decreased by 22 events, bleeding events by six events, and all ischaemic diagnoses by 37 events. This had the effect of decreasing the rate of stroke to 3.93/100 person years and increasing the RRs of the warfarin group when treated versus the non-warfarin group to 1.59 .

\section{DISCUSSION}

This retrospective study of a large UK population compared the risk of serious thromboembolic and bleeding events and survival in patients with NVAF treated with or without warfarin. Survival was increased in patients treated with warfarin, and this was related to the proportion of time they spent within the target INR range (2.0-3.0). Warfarin treatment was found to be associated with a lower risk of ischaemic and thromboembolic events and an increase in bleeding events.

The rate of stroke was significantly lower among patients treated with warfarin than among patients who were not treated with warfarin. However, the risk reduction observed $(26 \%)$ was substantially lower than in clinical trials where reductions in risk of stroke of up to two thirds have been reported. ${ }^{4-10}$ This suggests that in routine medical management such reductions are not achieved. The level of INR 
control may be an important factor in explaining this discrepancy. The rate of stroke observed in both groups is high relative to previous reports..$^{22-24}$ The requirement for a hospitalisation in the selection criteria may have biased the study population towards patients with more severe NVAF and a greater risk of stroke. If patients were treated according to guidelines, it would be expected that patients at greater risk would be selected for treatment. ${ }^{25}$ It is therefore unlikely that the lower stroke rate in the warfarin group is explained by this group being at lower initial risk than the non-warfarin group. This is supported by the very similar risk of stroke and all ischaemic or embolic events seen in the untreated group and in the treated group while not receiving warfarin.

The results show that the mean survival of patients treated with warfarin was more than a year longer than that of the group who did not receive warfarin. This survival advantage was maintained after controlling for age and for morbidity status. For morbidity status, days in hospital in the year before the NVAF index event was used. This was a convenient measure but we acknowledge that it is limited, as extent of morbidity may not necessarily be measured by length of stay.

The differences in survival are apparent even in the oldest patients. These data may indicate that a failure to treat patients with warfarin is associated with decreased survival. It has previously been shown that patients whose warfarin treatment is well controlled have a lower risk of thromboembolic events, in particular stroke. ${ }^{17}{ }^{26}$ In this study, the survival of patients with NVAF treated with warfarin was stratified by the quality of the INR control based on the proportion of time spent in the target INR range 2.0-3.0. In routine clinical practice the time individual patients spend in this target range varies considerably. ${ }^{25}$ Patients with well controlled INR survived on average more than a year and a half longer than patients with poor control, whose survival was indistinguishable from the non-warfarin treatment group. Although this difference in survival may be influenced by factors outside the dataset, it is consistent with previous reports that mortality is increased in warfarin treated patients with NVAF when their INR is outside the target range. ${ }^{15}$

This study confirmed previous observations that many patients with NVAF remain untreated. ${ }^{12}$ In this UK study population, only $36 \%$ of patients $(27 \%$ of the elderly $>75$ years) were treated with warfarin over the study period, and even these patients were treated for only $70 \%$ of the possible time from NVAF diagnosis. Several factors may have influenced this apparent undertreatment. Patients who were not treated with warfarin may have been at low risk and appropriately treated with aspirin. However, this is not consistent with the patterns observed in this study, where the proportion of patients treated with warfarin did not increase with increasing age. Only $26 \%$ of women older than 75 years, a group known to be at high risk, ${ }^{27}$ were treated with warfarin. The risk of all thromboembolic events is very similar for the non-warfarin group and the warfarin group when not treated. Reluctance by the physician or patient to accept the risk of adverse events associated with warfarin is known to contribute to undertreatment. ${ }^{16}$ The rate of hospital admission due to bleeding was lower in the warfarin group while untreated than in the non-warfarin treated group, suggesting that the non-warfarin group included patients at a higher initial risk of bleeding. This may have influenced the decision not to treat some of the patients with NVAF in this study. A physician would also be more reluctant to treat any patients who were already receiving medication that may interact with warfarin. ${ }^{16}$ The non-warfarin group was older than the warfarin group and the older patients may have been considered unable to cope with the regular INR monitoring required during warfarin treatment. Although some patients should not be treated with warfarin because of safety concerns, many patients within the untreated group probably would benefit from warfarin treatment.

The study design has some limitations. No data were available directly describing medication with warfarin and aspirin. Because of this, we assumed that only patients who had some degree of continuous monitoring (more than four INR tests) were treated with warfarin. Some of the patients with one to four tests may have discontinued treatment very early on. Further, only patients who had an inpatient diagnosis or record of NVAF were included. Patients examined in primary care and with no hospital admission relating to NVAF were excluded, and therefore the patients included in the study may be predicted to have had greater morbidity than a sample selected from both hospital and primary care. These morbidities may also include alcoholism, dementia, or other diseases that may preclude anticoagulation. There are methodological issues when attempting to estimate the proportion of time a patient is within or outside the INR target range. However, linear interpolation has also been shown to be the most valid and simplest technique compared with other methods. ${ }^{28}$ Given these limitations, these data describe the care and monitoring of a large cohort of patients, with a very large number of INR values, events, and deaths, and over a lengthy study period. Systematic biases-for example, coding inaccuracies-would be common to all comparative groups.

These data suggest that the failure to treat according to national guidelines in routine clinical practice, in terms both of patients selected for anticoagulation and of difficulty maintaining those selected patients within the recommended INR target range of 2.0-3.0, was associated with additional mortality and morbidity among patients with NVAF.

\section{Authors' affiliations \\ C J Currie, C L Morgan, School of Medicine, Cardiff University, Cardiff, UK \\ M Jones, P McEwan, School of Mathematics, Cardiff University, Cardiff, UK \\ J Goodfellow, Wales Heart Research Institute, Cardiff University, \\ Cardiff, UK \\ C Emmas, Outcomes Research AstraZeneca UK Ltd, Luton, UK \\ J R Peters, Department of Medicine, University Hospital of Wales, Cardiff, UK}

The study was funded by AstraZeneca (UK), which developed a product that will compete directly with warfarin for some indications including non-valvar atrial fibrillation. Their input was minimal, although they reviewed an almost finalised manuscript and made acceptable presentational changes.

\section{REFERENCES}

1 Go AS, Hylek EM, Phillips KA, et al. Prevalence of diagnosed atrial fibrillation in adults: national implications for rhythm management and stroke prevention: the anticoagulation and risk factors in atrial fibrillation (ATRIA) study. JAMA 2001;285:2370-5

2 Benjamin EJ, Wolf PA, D'Agostino RB, et al. Impact of atrial fibrillation on the risk of death: the Framingham heart study. Circulation 1998;98:946-52.

3 Wolf PA, Abbott RD, Kannel WB. Atrial fibrillation as an independent risk factor for stroke: the Framingham study. Stroke 1991;22:983-8.

4 The Stroke Prevention in Atrial Fibrillation Investigators. Stroke prevention in atrial fibrillation study: final results. Circulation 1991;84:527-39.

5 Connolly SJ, Laupacis A, Gent $M$, et al. Canadian atrial fibrillation anticoagulation (CAFA) 7 study. J Am Coll Cardiol 1991;18:349-55.

6 Petersen P, Boysen G, Godtfredsen J, et al. Placebo-controlled, randomised trial of anticoagulation and aspirin for prevention of thromboembolic complications in chronic atrial fibrillation. Lancet, 1989:i, 175-9.

7 The Boston Area Anticoagulation Trial for Atrial Fibrillation Investigators. The effect of low-dose anticoagulation on the risk of stroke in patients with nonrheumatic atrial fibrillation. N Engl J Med 1990;323:1505-11.

8 EAFT (European Atrial Fibrillation Trial) Study Group. Secondary prevention in non-rheumatic atrial fibrillation after transient ischaemic attack or minor stroke. Lancet 1993;342:1255-62.

9 Ezekowitz MD, Bridgers SL, James KE, et al. Anticoagulation in the prevention of stroke associated with nonrheumatic atrial fibrillation: Veterans Affairs Stroke Prevention in Nonrheumatic Atrial Fibrillation Investigators. NEng/JMed 1992;327:1406-12. 
10 Atrial Fibrillation Investigators. Risk factors for stroke and efficacy of antithrombotic therapy in atrial fibrillation: analysis of pooled data from five randomized controlled trials. Arch Intern Med 1994;154:1449-57.

11 Bungard TJ, Ghali WA, Teo KK, et al. Why do patients with atrial fibrillation not receive anticoagulation? Arch Intern Med 2000;60:41-6.

12 Ruigomez A, Johansson S, Wallander M-A, et al. Incidence of chronic atrial fibrillation in general practice and its treatment pattern. J Clin Epidemiol 2002;55:358-63.

13 Department of Health. National framework for older people. Standard 5: Stroke. London: Stationery Office, 2001.

14 Odén A, Fahlén M. Oral anticoagulation and risk of death: a medical record linkage study. BMJ 2002;325:1073-5.

15 Hylek EM, Go AS, Chang Y, et al. Effect of intensity of oral anticoagulation on stroke severity and mortality in atrial fibrillation. N Engl J Med 2003;349:1019-26.

16 Sudlow M, Thomson R, Thwaites B, et al. Prevalence of atrial fibrillation and eligibility for anticoagulants in the community. Lancet 1998;352:167-71.

17 Jones C, McEwan P, Morgan CL, et al. Evaluation of the pattern of treatment, level of anticoagulation control, and outcome of treatment with warfarin in subjects with non-valvar atrial fibrillation: a record linkage study in a large British population. Heart 2005;91:472-7

18 Gill L, Goldacre M, Simmons H, et al. Computerised linking of medical records: methodological guidelines. J Epidemiol Community Health 1993:47:316-9.

19 Rosendaal FR, Cannegieter SC, van der Meer FJM, et al. A method to determine the optimal intensity of oral anticoagulant therapy. Thromb Haemost 1993:69:236-9.
20 Currie CJ, Morgan CL, Gill L, et al. The epidemiology and costs of acute hospital care for cerebrovascular disease in diabetic and non-diabetic populations. Stroke 1997;28:1142-6.

21 Currie CJ, Morgan CL, Peters JR. Patterns and costs hospital care for coronary heart disease related and not related to diabetes. Heart 1997;78:544-9.

22 Wang TJ, Massaro JM, Levy DL, et al. A risk score for predicting stroke or death in individuals with new-onset atrial fibrillation in the community. JAMA 2003:290:1049-56.

23 Go AS, Hylek EM, Chang Y, et al. Anticoagulation therapy for stroke prevention in atrial fibrillation: how well do randomised trials translate into clinical practice? JAMA 2003;290:2685-92.

24 Kalra L, Yu G, Perez I, et al. Prospective cohort study to determine if trial efficacy of anticoagulation for stroke prevention in atrial fibrillation translates into clinical effectiveness. BMJ 2000;320:1236-9.

25 Baglin TP, Rose PE. Guidelines on oral anticoagulation: third edition. Br J Haematol 1998;101:374-87.

26 Jones M, McEwan P, Peters JR, et al. Anticoagulation with warfarin in patients with atrial fibrillation: a record linkage study of control and outcomes in a UK population [poster presentation]. Annual Meeting of the British Society for Haemostasis and Thrombosis (BSHT), Cambridge, UK, 17-19 September, 2003.

27 Hart RG, Pearce LA, McBride R, et al. Factors associated with ischemic stroke during aspirin therapy in atrial fibrillation. Stroke 1999;30:1223-9.

28 Hutten BA, Prins MH, Redekop WK, et al. Comparison of three methods to assess therapeutic quality control of treatment with vitamin $\mathrm{K}$ antagonists. Thromb Haemost 1999:82:1260-3.

\section{IMAGES IN CARDIOLOGY}

\section{Abnormal left atrial membranous structure in transthoracic echocardiography caused by external compression from a large bronchogenic cyst}

A 44 year old woman was referred for echocardiographic examination because of recent onset of dyspnoea. She had been diagnosed with bronchial asthma by a private clinic. On physical examination, however, pulmonary auscultation revealed bilateral vesicular breath sounds without rales and good heart sounds without murmur. Transthoracic echocardiography demonstrated a thin walled membranous structure dividing the left atrium. Both sides had the same echo density, indicating fluid density. Several possible diagnoses were initially possible, such as cor triatriatum, left atrial dissection, or an external mass. Colour Doppler investigation showed no communication between the sides of the abnormal membrane. Contrast echo using perfluorocarbon-exposed sonicated dextrose albumin showed no filling of contrast into the upper side of the membrane (panel A). There was no evidence of pulmonary arterial hypertension. Subsequent transoesophageal echocardiography (TOE) revealed a non-movable membranous flap in the upper portion of the left atrium. The TOE also indicated there was no flow communication across the flap. Cardiac magnetic resonance imaging showed a round contoured extracardiac mass about $5.5 \mathrm{~cm}$ in diameter that originated from the subcarina and compressed against the left atrium. The mass had a uniformly low signal on $\mathrm{Tl}$

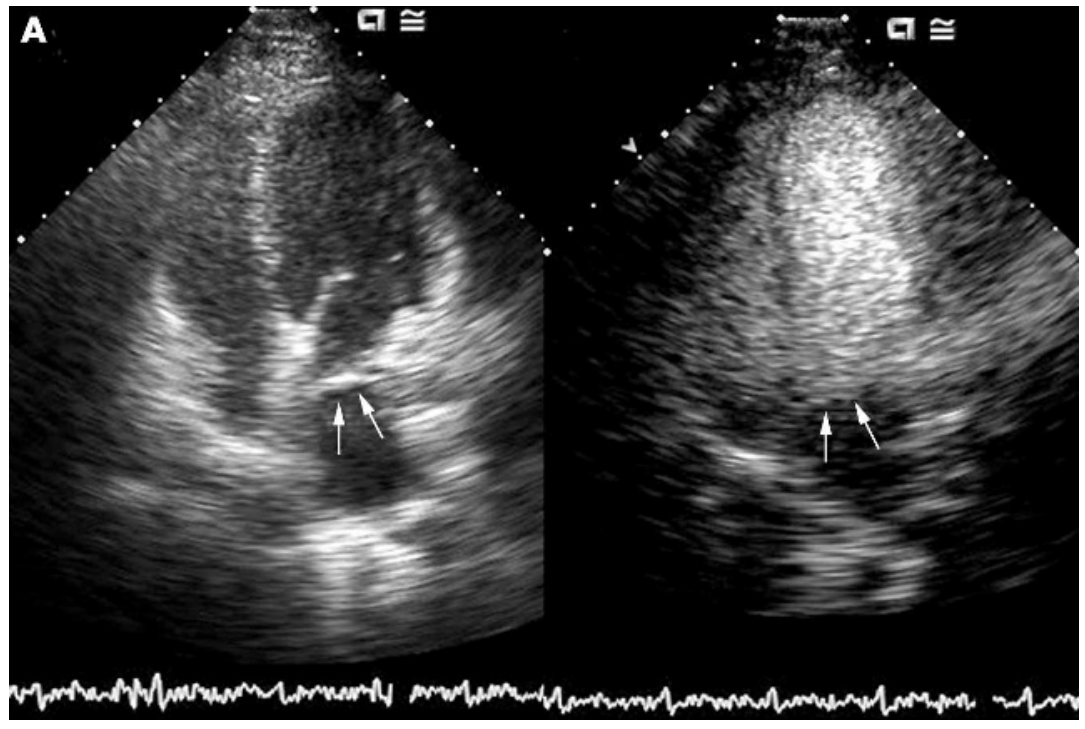

weighted image and a high signal on T2 weighted image without contrast enhancement (panel B). The mass was completely resected without complication and pathology confirmed it to be a bronchogenic cyst. The patient remains asymptomatic and there is no evidence of recurrence six months after surgery.

S-G Ahn

S-J Tahk J-H Shin shinjh@ajou.ac.kr 